\title{
Os desafios da oncologia: Da formação à ação profissional do enfermeiro
}

\author{
The challenges of oncology: From training to professional action of nurses \\ Los retos de la oncología: De la formación a la acción profesional de las enfermeras
}

Recebido: 20/05/2021 | Revisado: 28/05/2021 | Aceito: 02/06/2021 | Publicado: 18/06/2021

\author{
Rubiane Beal \\ ORCID: https://orcid.org/0000-0002-7323-732X \\ Faculdades Pequeno Príncipe, Brasil \\ E-mail: ruby3beal@hotmail.com \\ Kristian Sbolli \\ ORCID: https://orcid.org/0000-0001-9427-5125 \\ Faculdades Pequeno Príncipe, Brasil \\ E-mail: kristian.sbolli@hotmail.com \\ Elaine Rossi Ribeiro \\ ORCID: https://orcid.org/0000-0003-3492-217X \\ Faculdades Pequeno Príncipe, Brasil \\ E-mail: elaine.rossi@hotmail.com \\ Maria Rosa Machado Prado \\ ORCID: https://orcid.org/0000-0002-9795-3777 \\ Faculdades Pequeno Príncipe, Brasil \\ E-mail: mrosaprado@hotmail.com
}

\begin{abstract}
Resumo
Objetivo: Conhecer a percepção dos egressos de enfermagem sobre a formação acadêmica para o trabalho na área de oncologia. Método: Pesquisa exploratória, abordagem qualitativa, desenvolvida em uma Instituição de Alta Complexidade em oncologia na região Oeste do Paraná. A coleta de dados ocorreu de abril a maio de 2019, por meio de entrevistas semiestruturadas, com 35 enfermeiros atuantes na assistência de pacientes oncológicos. Foi realizada análise de conteúdo temática dos dados. Resultados: Foram elaboradas três categorias: Desafios na Oncologia, Formação para trabalhar em Oncologia e Especialização em Oncologia. Os enfermeiros apontaram dificuldades para a realização desse cuidado e a falta de contato com a temática no decorrer da graduação, buscando a especialização para suporte ao conhecimento. Os entrevistados propõem como o tema deve ser abordado na matriz curricular. Conclusão: É necessária a ampliação da discussão sobre oncologia durante a graduação do enfermeiro. Incluir o resumo.
\end{abstract}

Palavras-chave: Oncologia; Ensino; Enfermagem.

\begin{abstract}
Objective: To know the perception of nursing graduates about training for work in the area of oncology. Method: Exploratory research, qualitative approach, developed in an Institution of High Complexity in oncology in the West region of Paraná. Data collection took place from April to May 2019, through semi-structured interviews, with 35 nurses being employed in the care of cancer patients. Thematic data analysis was carried out. Results: Three thematic units were elaborated: Challenges in Oncology, Training to operate in Oncology and specialization in Oncology. The nurses pointed out the difficulties to perform this care and the lack of contact with the theme during graduation, seeking specialization to support knowledge. Interviewees' respondents propose how the topic should be addressed in the curriculum. Conclusion: It is necessary in order to expand the discussion on oncology during the nurse's graduation.
\end{abstract}

Keywords: Oncology; Teaching; Nursing.

\section{Resumen}

Objetivo: Conocer la percepción de los graduados en enfermería sobre la formación para el trabajo en el área de oncología. Método: Investigación exploratoria, enfoque cualitativo, desarrollada en una institución de alta complejidad en oncología en la región oeste de Paraná. La recopilación de datos tuvo lugar de abril a mayo de 2019, a través de entrevistas semiestructuradas, con 35 enfermeras que trabajan en la atención de pacientes con cáncer. Se realizó un análisis de datos temáticos. Resultados: se elaboraron tres unidades temáticas: desafíos en oncología, capacitación para trabajar en oncología y especialización en oncología. Las enfermeras señalaron dificultades para llevar a cabo esta atención y la falta de contacto con el tema durante la graduación, buscando especialización para apoyar el conocimiento. Los encuestados proponen cómo se debe abordar el tema en el plan de estudios. Conclusión: es necesario ampliar la discusión sobre oncología durante la graduación de la enfermera.

Palabras clave: Oncología; Ensenãnza; Enfermería. 


\section{Introdução}

O câncer é uma enfermidade que atinge a população do mundo e é uma das quatro principais causas de morte precoce em adultos menores de 70 anos de idade e crianças. Essa incidência vem aumentando no mundo devido envelhecimento e crescimento populacional, mas também pela mudança na distribuição e na prevalência dos fatores causadores de câncer (Bray et al., 2018).

A estimativa no Brasil aponta que ocorrerão 625 mil casos de câncer na população entre 2020-2022, exceto câncer de pele não melanoma (Ministério da Saúde, 2019). E um terço desses novos casos poderiam sem evitados pela redução ou eliminação dos fatores de risco ambientais e relacionados aos hábitos de vida. E as principais metas do tratamento são a cura, prolongamento da vida e melhora da qualidade de vida.

Dentro da equipe de profissionais da saúde que atuam na oncologia, os enfermeiros desempenham um papel essencial no apoio aos pacientes com câncer como: gerenciar complicações relacionadas à doença, monitorar as respostas dos pacientes à intervenções realizadas e coordenar o atendimento aos pacientes (Gilbert et al., 2011).

A atuação dos profissionais de enfermagem em oncologia demanda habilidades de cuidados de alta complexidade durante todo o processo terapêutico, exigindo dos enfermeiros extrema habilidade relacional e afetiva, considerando as necessidades individuais de cada um (Lins \& Souza, 2018).

A prática de enfermagem na oncologia abrange todos os grupos etários e é realizada em diversos ambientes de cuidados em saúde como no domicílio, na comunidade, nas instituições de cuidados agudos e nos centros de reabilitação.

E a boa prática assistencial só é possível com formação adequada abrangendo todas as linhas do cuidado. Portanto, A preocupação na formação destes profissionais fez com que a Resolução n 3/2001 do Conselho Nacional de Educação passasse a determinar, em seu artigo $3^{\circ}$, do Curso de Graduação em Enfermagem, que o perfil do enfermeiro formado contemple uma atuação generalista, crítica e reflexiva que seja capaz de conhecer e intervir sobre os problemas/situações de saúde-doença mais prevalentes no perfil epidemiológico nacional, com ênfase na sua região de atuação, identificando as dimensões biopsico-sociais dos seus determinantes (Resolução CNE/CES nº 3, 2001 ).

Mesmo com um direcionamento generalista na formação do enfermeiro, observa-se que, no preparo desse profissional existem lacunas em algumas áreas específicas como na oncologia. Constata-se carência de conhecimentos desde conceitos básicos até específicos dessa área, reportando à necessidade de conhecer como as matrizes curriculares da formação do enfermeiro trabalham os conteúdos voltados para a oncologia como preconizado nas diretrizes curriculares (Luz, Vargas, Rosa, \& Schmitt, 2016).

Diante do problema, esta pesquisa teve como questão norteadora: Como os enfermeiros estão sendo preparados para atuar na área de oncologia? E objetivou-se conhecer a percepção dos egressos de enfermagem sobre a formação para o trabalho na área de oncologia.

\section{Metodologia}

Trata-se de uma pesquisa exploratória com abordagem qualitativa. As pesquisas qualitativas trabalham com objetos que correspondem a um espaço profundo das relações, dos processos e dos fenômenos que não podem ser reduzidos à operacionalização de variáveis. Este tipo de pesquisa permite revelar processos sociais, ainda pouco conhecidos, referentes a grupos particulares, fazer revisão e criação de novos conceitos e categorias durante a criação (Minayo, 2014). Já as pesquisas exploratórias buscam proporcionar uma visão geral de determinado fenômeno.

Esta pesquisa foi realizada em um serviço de oncologia de alta complexidade hospitalar diante do cuidado de paciente com câncer, localizada na região Oeste do estado do Paraná. Participaram 35 enfermeiros que trabalham em unidade de 
internação e ambulatório hospitalar de quimioterapia. A coleta de dados ocorreu no período de janeiro a março de 2019, por meio de entrevista semiestruturada com questionário de perguntas abertas, nas quais o entrevistado teve a possibilidade de escrever sobre a percepção em relação a sua formação para o trabalho na área de oncologia, respondendo perguntas que versavam a respeito de: primeiro contato com pacientes em tratamento oncológico; conhecimentos e habilidades para trabalhar em oncologia após a graduação de enfermagem; os maiores desafios encontrados na atuação em Oncologia; a contribuição da matriz curricular do curso de enfermagem para atuação em oncologia; como deve ser a formação do enfermeiro para atuar em Oncologia; já fez especialização na área de oncologia e o que a especialização agregou a vida profissional, bem como o motivo para procurar especialização na área de oncologia.

As entrevistas, foram realizadas pela primeira autora do artigo e aconteceram em data e horário agendados, segundo a disponibilidade dos participantes, e para garantir a privacidade do mesmo as entrevistas foram realizadas em local privado.

As entrevistas foram transcritas na íntegra e analisadas seguindo-se as três etapas da análise de conteúdo temática de Minayo (2014), primeira etapa é a pré-análise com leitura flutuante para conhecer o conteúdo do material empírico gerado pelas entrevistas, a segunda etapa é a exploração do material onde é realizada a operação classificatória com o intuito de alcançar o núcleo de compreensão do texto e estabelecer as categorias e a terceira etapa é o tratamento e a interpretação dos resultados, na qual é possível fazer inferências à luz da literatura científica sobre o ensino de oncologia na percepção dos enfermeiros.

A pesquisa foi aprovada pelo Comitê de Ética e Pesquisa sob o número 3.216.164. Após autorização do Comitê de Ética foi realizado contato com os enfermeiros na própria Instituição hospitalar cenário de estudo e o aceite foi formalizado com a assinatura do Termo de Consentimento Livre Esclarecido, foi mantido o anonimato dos participantes os quais receberam códigos numéricos em ordem sequencial precedidos da abreviatura "Enf" referente a enfermeiro.

\section{Resultados e Discussão}

\subsection{Perfil sociodemográfico}

Os dados do perfil sóciodemográfico estão representados por meio de números absolutos e porcentagens até a segunda casa decimal.

Em relação à distribuição dos participantes, de acordo com dados do Lins e Souza (2018), o maior número de enfermeiros é composto pelo sexo feminino. A equipe de enfermagem no Paraná é predominantemente feminina, sendo constituída por $87,9 \%$ de mulheres, no entanto, mesmo em se tratando de uma categoria feminina registra-se a presença de 11,9\% dos homens, portanto abaixo da média nacional que é de 14,4\%. Na Tabela 1 observa-se a predominância do sexo feminino no estudo realizado.

Tabela 1 - Distribuição dos participantes de acordo com o sexo.

\begin{tabular}{ccc}
\hline Sexo & N & \% \\
\hline Feminino & 27 & $77,14 \%$ \\
Masculino & 08 & $22,85 \%$ \\
& $\mathbf{3 5}$ & $\mathbf{1 0 0 \%}$ \\
\hline
\end{tabular}

Fonte: Autores (2021).

Na Tabela 2 destaca-se que a pouca idade se constitui como um obstáculo enfrentado pelos egressos no início de suas 
atividades profissionais, pois essa pouca idade reflete a falta de experiência de vida, bem como inabilidade para lidar com experiências às vezes dolorosas.

Tabela 2 - Distribuição dos participantes de acordo com a idade.

\begin{tabular}{ccc}
\hline Idade & N & \% \\
\hline 22 a 26 anos & 12 & $34,28 \%$ \\
27 a 31 anos & 9 & $25,72 \%$ \\
32 a 36 anos & 8 & $22,86 \%$ \\
37 a 41 anos & 3 & $8,57 \%$ \\
42 a 46 anos & 3 & $8,57 \%$ \\
Total & $\mathbf{3 5}$ & $\mathbf{1 0 0 \%}$ \\
\hline
\end{tabular}

Fonte: Autores (2021).

Constata-se na Tabela 3, que apenas 5,71\% dos participantes possuem especialização em oncologia, fato que chama a atenção, uma vez que o paciente oncológico necessita de profissionais capacitados para prestar assistência integral alcançando as reais necessidades de atenção e cuidados específicos.

Tabela 3 - Distribuição dos participantes conforme a especialidade.

\begin{tabular}{ccc}
\hline Especialização & $\mathbf{N}$ & $\mathbf{\%}$ \\
\hline Oncologia & 2 & $5,71 \%$ \\
Nefrologia & 1 & $2,88 \%$ \\
Saúde pública & 4 & $11,42 \%$ \\
Urg. E Emergência & 4 & $11,42 \%$ \\
Em andamento & 5 & $14,29 \%$ \\
Não tem & 19 & $54,28 \%$ \\
Total & $\mathbf{3 5}$ & $\mathbf{1 0 0 \%}$ \\
\hline
\end{tabular}

Fonte: Autores (2021).

\subsection{Percepção dos participantes sobre a formação para o trabalho na área de oncologia}

Por meio da análise das respostas dos enfermeiros entrevistados foram elaboradas três categorias: Os desafios da Oncologia; Formação para Trabalhar em Oncologia e Especialização em Oncologia como demonstrado no Quadro1. 
Quadro 1 - Distribuição das categorias.

\begin{tabular}{|c|c|c|}
\hline Categorias & Subcategorias & Unidades de contexto \\
\hline Desafios na Oncologia & $\begin{array}{l}\text { - Primeiro contato com oncologia } \\
\text { - Atuação em oncologia }\end{array}$ & $\begin{array}{c}\text { - Emprego } \\
\text { - Estágio } \\
\text { - Preparo técnico } \\
\text { - Preparo emocional }\end{array}$ \\
\hline $\begin{array}{c}\text { Formação para } \\
\text { Trabalhar em Oncologia }\end{array}$ & $\begin{array}{c}\text { - Apto em conhecimentos e } \\
\text { habilidades } \\
\text { - Matriz curricular } \\
\text { - Como deve ser formação }\end{array}$ & \\
\hline $\begin{array}{l}\text { Especialização em } \\
\text { Oncologia }\end{array}$ & $\begin{array}{c}\text { - Motivação para buscar } \\
\text { especialização } \\
\text { - Conhecimentos adquiridos com } \\
\text { a especialização }\end{array}$ & \\
\hline
\end{tabular}

Fonte: Autores (2021).

A subcategoria "Primeiro contato com oncologia", permitiu apreender sobre a experiência dos participantes em relação ao seu primeiro contato com pacientes oncológicos, durante os estágios extracurriculares da graduação e no primeiro emprego. Assim demonstram-se as respostas nos discursos a seguir:

Meu primeiro emprego foi em um hospital oncológico (Enf $\left.n^{\circ} 35\right)$.

No hospital oncológico, assim que contratada (Enf $\left.n f^{\circ} 8\right)$.

Foi possível observar que $20 \%$ dos participantes relataram que tiveram contato prévio com a oncologia durante os estágios extracurriculares do Curso de Enfermagem em unidade de saúde pública e hospitalar. Eles descreveram esse contato nos seguintes discursos:

Meu primeiro contato foi nos estágios de formação onde passamos por Hospital Oncológico (Enf n $n^{\circ}$ 2).

Ano de 2014, estágio pela instituição, primeiro contato visual do tratamento do paciente, observando condutas" (Enf $\left.n^{\circ} 9\right)$

Em 2015 quando meu pai foi diagnosticado com câncer de estomago. E após há 3 anos atrás quando fui contratada para trabalhar na instituição atual (Enf $\left.n^{\circ} 3\right)$.

Estudo de Lins e Souza (2018), constata-se que os participantes encontraram dificuldades na assistência de enfermagem aos pacientes durante a graduação e 42,5\% identificaram que o período de estágio é curto.

A prática de um estágio na área da saúde é algo comum e precisa ser articulado à formação do aluno com a atuação profissional permitindo que os acadêmicos vivenciem as experiências profissionais contribui para a formação técnica e humana no relacionamento com os pacientes.

A realização de estágios extracurriculares em diferentes especialidades de instituições hospitalares auxilia à formação técnica e faz o aluno vivenciar realidades profissionais, muitas vezes diferentes oferecendo-lhes a oportunidade de visualizar as particularidades de cada instituição, bem como as de cada área de atendimento de enfermagem.

Na unidade de contexto "Atuação em Oncologia", os participantes relatam sobre as dificuldades e adaptações ao 
assumir sua formação como enfermeiro no momento em que se depararam com situações a serem enfrentadas, diante de pacientes oncológicos em relação ao preparo técnico e emocional como observado nos relatos a seguir:

Para um recém formado como eu foi tudo, pois tive que aprender desde o funcionamento até a prática para obter conhecimento (Enf $\left.n^{\circ} 33\right)$.

Lidar com a dor, câncer causa um desgaste físico e mental muito grande no doente, e isso causa muitas vezes um despreparo emocional para o profissional $\left(E n f n^{\circ} 9\right)$.

Acredito que seja a dor do paciente e os desafios que ele passa durante o tratamento (Enf $\left.n^{\circ} 11\right)$.

Separar o profissional do emocional, e a particularidade de cada medicação e quimioterapia (Enf $\left.n^{\circ} 13\right)$.

Foi encontrado nos relatos a existência de dificuldades por parte do profissional de se desvincular do emocional diante dos problemas vivenciados junto aos pacientes. A atenção oncológica coloca os profissionais em contato estreito com situação de dor, finitude e morte, além dos efeitos colaterais que desencadeiam graves reações físicas e emocionais, desesperança de pacientes e familiares, bem como a expectativa de cura da doença. Esses elementos imputam, aos profissionais, a necessidade de enfrentamentos perenizados durante a operacionalização da assistência aos usuários (Lins, \& Souza, 2018). Kirby et al. (2020) destacam que a prática da oncologia apesar de gratificante, demanda do profissional preparo não apenas técnico científico, mas também emocional, para enfrentar a rotina do diagnóstico, tratamento e prognóstico do paciente diagnosticado com câncer e seus familiares e cuidadores.

Quando falamos em enfermagem na oncologia atribuímos fases de assistência relacionadas ao tratamento oncológico como sinais e sintomas para o diagnóstico do câncer, tratamento com terapia antineoplásica, oncologia clínica, oncologia cirúrgica e os cuidados no final de vida. Esses cuidados exigem conhecimentos, habilidades e atitudes por parte dos profissionais de enfermagem durante o acompanhamento de pacientes adultos e crianças. Observou-se que os participantes ao falarem sobre o cuidado em crianças em tratamento oncológico, relatam o quão difícil é a oncologia pediátrica, conforme relatos a seguir:

Conduzir oncologia em pediatria. E no setor clínico adulto, a conduta "paliativa" a aceitação dos familiares (Enf $n^{o}$ 3).

Maiores desafios é lidar com quimioterapia (protocolo) e com a pediatria, pois as crianças precisam de um cuidado redobrado. Também lidar com dispositivos como port a cath. São essas questões que na minha opinião, durante a graduação são falhas (Enfn $\left.{ }^{\circ} 2\right)$.

Nesses discursos desvela-se o total despreparo do enfermeiro para atuar em oncologia pediátrica, pois requer cuidados específicos e conhecimentos sobre os protocolos de quimioterapia, o qual é utilizado desde a fase inicial até a fase terminal com o tratamento de quimioterapia paliativa. A administração de quimioterapias é atividade privativa do enfermeiro que está à frente dos cuidados ao paciente em tratamento oncológico, portanto deve ter o entendimento de todo esse processo, desde a administração da quimioterapia até orientação a respeito dos cuidados para o paciente e para a família.

Observa-se nos discursos, que os enfermeiros não estão preparados para lidar com o processo de morte, visto que, durante a formação acadêmica o tema da morte é pouco abordado. Isso resulta em várias lacunas, sendo que o profissional é incentivado a acreditar que somente a cura e a recuperação do paciente são características de um bom cuidado (Silveira et al., 
2016). A enfermagem está em constante interação com os pacientes por passar a maior parte do tempo cuidando dos mesmos e está aproximação resulta em desgaste emocional.

O enfermeiro na oncologia precisa desempenhar um papel essencial na exploração permanente do cuidado e das necessidades de cada paciente com câncer - que recebe tratamento ambulatorial de curta duração e internações para tratamento de longa duração a fim de que possa planejar cuidados específicos da melhor maneira possível (Calvo \& Sepulveda-Carrillo, 2016).

A categoria "formação para trabalhar em oncologia" investigou os participantes sobre o conhecimento adquirido, após sua formação na graduação de enfermagem percebe-se como seria uma boa formação e os participantes relataram sobre a formação recebida durante a graduação e se foi apropriada para desempenhar suas atividades na atuação em oncologia.

$\mathrm{Na}$ Unidade de Contexto, "Apto em conhecimentos e habilidades" para atuar na oncologia, revelou-se que os enfermeiros consideram que não receberam formação suficiente para atuar e atender às demandas de cuidados com paciente oncológico. Os participantes trazem nos seus discursos que durante a formação o conteúdo ministrado com relação à oncologia foi insuficiente.

De forma alguma. Na graduação as informações sobre a matéria sobre oncologia fica muito vago e quase não é mencionado na grade. $O$ assunto é abordado durante a matéria sobre a saúde da mulher que engloba câncer de mama e útero $\left(E n f n^{\circ} 5\right)$.

As Diretrizes Curriculares Nacionais do curso de graduação em Enfermagem, Resolução No 03/2001, preconizam que as instituições de ensino formem um enfermeiro generalista, apto e com conhecimentos para atuar em diferentes áreas da saúde; os conteúdos essenciais para o Curso de graduação em Enfermagem devem estar relacionados com todo o processo saúde-doença do cidadão, da família e da comunidade, integrado à realidade epidemiológica e profissional, proporcionando a integralidade das ações do cuidar em enfermagem (Resolução CNE/CES nº 3, 2001 ).

Como consequência dessa falta de preparo e conhecimento, observa-se o despreparo dos enfermeiros para atuar na oncologia. O cuidado de enfermagem ao paciente oncológico deve ser realizado de maneira holística, mas muitas vezes esse cuidado não é oferecido, devido à falta de conhecimento com respeito às ações do cuidado por parte dos profissionais de saúde, o que pode interferir no tratamento e recuperação do paciente (Novaes et al., 2016).

Um dos enfermeiros relatou que acreditava estar apto para atuar na oncologia, porém ao se deparar com o dia a dia de um hospital oncológico sentiu dificuldade pela falta de conhecimento sobre o assunto.

No início nos consideramos aptos em conhecimentos e habilidade, porém durante o atendimento a beira leito, acabamos nos deparando com dificuldades inesperadas devido a área de oncologia ser ampla e durante a grade curricular "aprendemos" ou nos é passado os conhecimentos básicos somente (Enf 31).

Foi possível detectar que existe déficit de conhecimento em oncologia durante a formação, o que dificulta que esse profissional possa se identificar com a área da oncologia durante a atuação. A necessidade social de atuação competente por parte dos diversos profissionais da área da saúde, que devem considerar a incidência atual da doença, os altos índices de incidência e prevalência esperados para as próximas décadas (Luz, Vargas, Rosa, et al., 2016).

Contudo, no Art. $7^{\circ}$ da Resolução No $03 / 2001$, do Conselho Nacional de Educação, consta que na formação do enfermeiro, além dos conteúdos teóricos e práticos desenvolvidos ao longo de sua formação, ficam os cursos obrigados a incluir no currículo o estágio supervisionado em hospitais gerais e especializados, ambulatórios, rede básica de serviços de 
saúde e comunidades nos dois últimos semestres do curso de graduação em enfermagem (Resolução CNE/CES nº 3, 2001).

A unidade de contexto "Matriz Curricular", apreende-se que a área de oncologia para enfermagem é pouco ou quase não abordada, evidenciando-se como uma formação deficitária para atuar nessa área.

Não. Eu acredito que a grade curricular deveria ser melhor distribuída, para que pudéssemos ter o mínimo de conhecimento das variadas especialidades. E principalmente a oncologia, que é uma doença que atinge grande parte da população (Enf $\left.n^{\circ} 2\right)$.

A teoria quando está desvinculada de uma ação prática pode ser considerada estéril, pois limita a formação profissional para ter inserção na prática profissional. Em termos de exigência para a formação, $90 \%$ dos enfermeiros pesquisados indicaram que precisam de mais formação para o desempenho de suas atividades profissionais à atuação na área de oncologia (Ortega et al., 2015).

Um dos entrevistados ao relatar que a matriz curricular contribuiu pouco em sua formação, indaga sobre melhorias na qualificação profissional e reforçar sobre a necessidade do mercado de trabalho nos dias atuais com relação a profissionais habilitados para atuar na área de oncologia.

A grade curricular contribuiu pouco para a atuação, as instituições deveriam inserir a disciplina de oncologia visando na melhoria da capacitação e qualificação profissional ao se deparar com a necessidade no mercado de trabalho $\left(E n f n^{\circ} 31\right)$.

O ensino da oncologia aos estudantes de Enfermagem deve atrelar teoria e prática em estágios interprofissionais garantindo uma experiência compartilhada aos acadêmicos. O modelo de ensino proposto para lidar com o paciente oncológico deve ser centrado na pessoa, desde o momento da entrada no sistema na atenção básica à saúde até o fim da vida em situação hospitalar (Marques, 2019).

As Diretrizes Curriculares Nacionais para os cursos de graduação constituem orientações para elaborar currículos e devem ser obrigatoriamente adotadas por todas as instituições de Ensino Superior, assegurando, dentro de muitos aspectos a flexibilidade da formação e abandonando antigas concepções. Isso busca garantir formação básica sólida capaz de preparar o profissional para os desafios e as transformações rápidas da sociedade (Resolução CNE/CES nº 3, 2001).

A Unidade de Contexto, "Como deve ser a formação", os participantes relacionaram as necessidades de formação, no momento em que se deparam com as dificuldades na atuação em oncologia, explicitam que a graduação acadêmica deve ser repensada para melhorar a formação conforme discursos mencionados

Acredito que uma disciplina exclusiva que abranja todo o conceito de câncer, assim como cuidados e suporte paliativos $\left(\operatorname{Enf} n^{\circ} 7\right)$.

O câncer é uma doença com grande incidência de alta prevalência e mortalidade. São necessários diversos tipos de serviços de saúde e ações específicas. Portanto, é indispensável discutir sobre a formação de recursos humanos voltados ao cuidado do paciente oncológico em toda a linha de cuidado (Oliveira, Stancato, \& Silva, 2018).

Um dos participantes relata sobre o enfoque da sua formação ser em saúde pública: 
em saúde pública $\left(\operatorname{Enf} n^{\circ} 31\right)$.

Todos os enfermeiros que atuam na área hospitalar ou em saúde pública, provavelmente, terão contato com pacientes oncológicos e precisarão apresentar compreensão do câncer e seu tratamento, educação nos princípios básicos e na prática do câncer, cuidados habilidade de avaliar as necessidades de cuidados de pacientes e orientações aos familiares (Royal College of Nursing, 2017).

A assistência de enfermagem qualificada e eficaz requer a capacidade do profissional de lidar com seus próprios sentimentos perante o paciente com câncer, por isso é importante que o sofrimento gerado não influencie a prestação de cuidados ao paciente com câncer e sua família (Bubolz, 2019).

O profissional de enfermagem com visão crítica aos cuidados deve receber durante a formação universitária a compreensão sobre seu papel no gerenciamento, na assistência e na pesquisa, proporcionando meios de valorização profissional e a possibilidade de alcançar uma melhor formação e condições de trabalho.

Apesar das mudanças ocorridas nas propostas das políticas de educação e de saúde, a formação se faz presente nos cursos de graduação de Enfermagem, os quais muitas vezes, são voltados para cuidados centrados no paciente.

A categoria "Especialização em oncologia" permitiu extrair duas unidades de contexto, nas quais forma investigados o que levou o participante a procurar uma especialização.

A Unidade de Contexto "Motivação para buscar especialização", evidenciou-se que a maioria dos participantes atuante no hospital oncológico do referente estudo não possuíam especialização em oncologia, mas relatam a vontade de realizar, conforme discursos a seguir:

Não, porém tenho vontade de me aprofundar no conhecimento em oncologia (Enf $\left.n^{\circ} 2\right)$.

Até o presente momento não. Porém, pretendo faze-la $\left(E n f n^{\circ} 3\right)$.

Esses desafios na atuação em oncologia exigem cada vez mais trabalhadores qualificados e aperfeiçoados para lidar com as novas demandas do exercício profissional direcionadas à realidade epidemiológica do nosso país.

Diante da carência da formação enfermeiros procuram especialização na área de oncologia e observa-se nos relatos que revelam essa procura em função da própria necessidade de adquirir conhecimentos. Outro ponto observado é que não se ressalta o apoio das instituições hospitalares oncológicas para essa especialização.

Tudo isso reforça a essencialidade do ensino continuado de uma especialização até mesmo as próprias empresas oferecem cursos de educação continuada para que esse déficit de aprendizagem diminua (Souza, Maracaipe, Albuquerque, \&Almeida, 2019).

Diante da dificuldade de lidar com as pessoas com câncer os participantes deste estudo relatam quais os benefícios do aprimoramento profissional, conforme observado nos relatos a seguir:

Aprofundar o conhecimento, para prestar uma melhor assistência, conhecendo a doença e o paciente como um todo $\left(\operatorname{Enf} n^{\circ} 2\right)$

Para que eu possa me preparar melhor, afim de aperfeiçoar minhas condutas como enfermeira e conseguir motivar cada vez mais minha equipe, afim de concluirmos juntos quão delicado é prestar assistência ao paciente oncológico (Enf $\left.n^{\circ} 3\right)$. 
Acredito que, para compreender melhor as causas, formação e níveis/estágios do câncer onde podemos atuar de maneira mais eficaz e consciente no atendimento e suporte como um todo ao paciente (Enf $n^{\circ} 7$ ).

Diante da complexidade de cuidados ao paciente com câncer, tanto na esfera técnico-científica como na emocional, constata-se que os enfermeiros tenham consciência do seu despreparo para lidar com oncologia.

$\mathrm{O}$ enfermeiro após iniciar seu cuidado assistencial em oncologia e adaptar-se ao novo ambiente, evidencia o grande comprometimento e responsabilidade pela sua profissão e principalmente pelos seus pacientes e familiares. Isto é, a vivência é intensa a vinculação com a área e efetiva-se pela compreensão de que o cuidado vai além do biológico, superando o sofrimento e a penalização em direção a uma visão pautada no sentimento de gratificação pelo trabalho desenvolvido (Luz, Vargas, Barlem et al., 2016).

Na subcategoria "Conhecimentos adquiridos com a especialização", os participantes desmistificam os conhecimentos agregados com o curso, conforme relatos a seguir:

Agregou a parte técnica, principalmente na parte de quimioterapia $\left(\right.$ Enf $\left.n^{\circ} 11\right)$.

Conhecimento em tipos de tumores etc. reações a quimio, protocolos etc (Enf $\left.n^{\circ} 18\right)$.

Esta agregando em ter mais conhecimento, pois no dia-a-dia acabamos não buscando informações necessárias por falta de tempo durante expediente e a troca de informações e conhecimento com outros profissionais é essencial (Enf $\left.n^{\circ} 31\right)$.

A pós-graduação é um dos recursos procurados pelos enfermeiros, com possibilidade de formação específica na área e experiência significativa no campo de atuação, pois o conhecimento produzido por meio de pesquisas voltadas para o cuidado em oncologia propicia a esses profissionais um diferencial acerca dos cuidados construídos a partir do pensamento científico de suas ações (Santos, Camelo, Laus, \& Leal, 2015).

Diante dos relatos, os enfermeiros solicitam melhorias ao ensino de oncologia para a graduação de enfermagem. Observa-se a necessidade de ampliar o nível de conhecimento dos graduandos de enfermagem no campo da oncologia, por meio de incentivo à participação dos graduandos em programas de iniciação científica, extensão e promoção à saúde. Dessa forma, os futuros profissionais de enfermagem poderão se sentir mais seguros ao transmitir o conhecimento para a população envolvida, de certa forma, com a oncologia (Silveira et al., 2016).

\section{Considerações Finais}

Atendendo ao objetivo de conhecer a percepção de enfermeiros sobre a formação para trabalhar na área de oncologia, observou-se que os enfermeiros não se sentem preparados para atuar em oncologia após a formação.

Dessa maneira, podemos perceber que o ensino de oncologia é necessário e deve permear a formação acadêmica, incentivado os estudantes de enfermagem a serem participes na aquisição de conhecimentos, habilidades e atitudes no seu processo de ensino aprendizagem com o intuito de atuarem com excelência na área de oncologia.

Contudo este estudo mostra uma falha no ensino de oncologia para enfermagem, permitindo novas pesquisas na área de ensino sobre oncologia, para que possam ser tomadas medidas que contribuam para formação dos futuros profissionais de enfermagem fornecendo subsídios para atuação qualificada em conhecimentos para o atendimento de um paciente oncológico em toda sua totalidade.

O presente estudo ressalta a necessidade de pesquisas futuras sobre quais metodologias de ensino melhor se adequam 
no ensino de oncologia nos cursos de enfermagem e também é necessário investigar esse tema em outros cursos da área da saúde, uma vez que as equipes atuantes na área de oncologia são formadas por vários profissionais da saúde.

\section{Referências}

Bray, F., Ferlay, J., Soerjomataram, I., Siegel, R. L., Torre, L. A., \& Jemal, A. (2018). Global cancer statistics 2018: GLOBOCAN estimates of incidence and mortality worldwide for 36 cancers in 185 countries. CA Cancer Journal Clinicians, 68(6), 394-424.

Bubolz, B. K., Barboza, M. C., N., Amaral, D. E. D., Viegas, A. C., Bernardes, L. S, \& Muniz, R. M. (2019). Perceptions of nursing professional with regards to the suffering and its coping strategies in Oncology. Rev Fund Care, 11(03): 599-606.

Calvo, L. E. A, \& Sepulveda-Carrillo, G. J. (2016). Care needs of câncer patients undergoing ambulatory treatment. Revista Electrónica Trimestral de Enfermería, 16(1): 353-358.

Gilbert, J. E., Green, E., Lankshear, S., Hughes, E., Burkoski, V., \& Sawka, C. (2011). Nurses as patient navigators in cancer diagnosis: review, consultation and model design. European Journal of Cancer Care, 20(02), 228-236.

Lins, F.G., \& Souza, S.R. (2018). Formação dos enfermeiros para o cuidado em oncologia. Revista de Enfermagem - UFPE, 12(1), 66-74.

Luz, K. R., Vargas M. A. O, Barlem, E. L. D., Schmitt, P. H., Ramos, F. R. S., \& Meirelles, B. H. S. 2016. Estratégias de enfrentamento por enfermeiros da oncologia na alta complexidade. Revista Brasileira de Enfermagem, 69(1): 67-71.

Luz, K. R., Vargas, M. A. O., Rosa, L. M., \& Schmitt, PH. (2016). Enfermeiro na atenção oncológica: conhecimento na prática do cuidado. Revista de Enfermagem - UFPE, 10(9): 3369-3376.

Kirby, E. E. F., Jung, I. E. F. S., Neves, L. M. L., Gregório, A. P. A., \& Gouvêa, M. V. (2021). Síndrome de Burnout em profissionais que atuam na oncologia: Revisão integrativa. Research, Society and Developmen, 9(7): 1-23.

Marques, A. C. S. B. (2019). Competências e habilidades para o ensino da oncologia na graduação de enfermagem no Brasil. Dissertação de Mestrado, Faculdade de Medicina de Botucatu - FMB, Botucatu, SP, Brasil.

Minayo, M. C. S. (2014). O desafio do conhecimento: pesquisa qualitativa em saúde. (14a ed.). Hucitec.

Ministério da Saúde. (2019). Estimativa 2020 - Incidência de Câncer no Brasil [livro-versão eletrônica]. Rio de Janeiro: Instituto Nacional de Câncer José Alencar Gomes da Silva - INCA.

Novaes, N. B., Ferraz, R. R. N., Rodrigues, F. S. M., Errante, P. R., Barnabé, A. S., Fornari, J. V., Szamszoryk, M., \& Silva, R. N. (2016). Cuidados prestados a paciente oncológicos sob a percepção de graduandos de enfermagem. Science in Health, 7(1): 11-15.

Oliveira, A. M., Stancato K, \& Silva, E. M. (2018). Formação do enfermeiro: políticas públicas na atenção oncológica. Revista Enfermagem em Foco, 9(3): 48-52.

Ortega, M. C. B., Cecagno, D., Lior A. M. S., Siqueira, H. C. H., Montesinos, M. J. L., \& Soler, L. M. (2015). Formação acadêmica do profissional de enfermagem e sua adequação às atividades de trabalho. Revista Latino-Americana de Enfermagem, 23(3): 404-410.

Resolução CNE/CES n ${ }^{\circ}$ 3, de 7 de novembro de 2001 (2001). Institui Diretrizes Curriculares Nacionais do Curso de Graduação em Enfermagem. http://portal.mec.gov.br/cne/arquivos/pdf/CES03.pdf

Royal College of Nursing. (2017). Career and education framework for cancer nursing. [livreto-versão eletrônica]. Londres: Royal College of Nursing 20 Cavendish Square.

Santos, F., Camelo, S. H. E., Laus, A. M., \& Leal, L. A. (2015). O enfermeiro que atua em unidades hospitalares oncológicas: perfil e capacitação profissional. Revista Electrónica Trimestral de Enfermería, 38(1): 313-323.

Silveira, N. R., Nascimento E. R. P., Rosa L. M., Jung, W., Martins S.R., \& Fontes M. S. (2016). Palliative care and the intensive care nurses: feelings that endure. Revista Brasileira de Enfermagem, 69(6): 1012-1019.

Souza, G. S. B., Maracaipe, L. F., Albuquerque, I. A., \& Almeida, A. B. (2019). Processo de formação do enfermeiro na prática oncopediátrica. Revista de Iniciação Científica e Extensão, 2(1): 46-50. 